\title{
Hemangioma intraósseo difuso agressivo: Relato de caso
}

\section{Aggressive Diffuse Intraosseous Hemangioma: Case Report}

\author{
Monique Alves ${ }^{10} \quad$ Marcus Sofia Ziegler ${ }^{1(1)}$ Fernando Augusto Dannebrock ${ }^{1}$ (1) \\ Erasmo de Abreu Zardo ${ }^{10}$ Carlos Marcelo Donazar Severo ${ }^{1}{ }^{\circledR}$ \\ ${ }^{1}$ Serviço de Ortopedia e Traumatologia, Hospital São Lucas, Pontifícia
Universidade Católica do Rio Grande do Sul, Porto Alegre, RS, Brasil \\ Rev Bras Ortop \\ Endereço para correspondência Monique Alves, Serviço de \\ Ortopedia e Traumatologia, Pontifícia Universidade Católica do Rio \\ Grande do Sul, Av. Ipiranga, 6690, Jardim Botânico, Porto Alegre, RS, \\ 90610-000, Brasil (e-mail: ma.moniquealves@gmail.com).
}

\section{Resumo \\ Palavras-chave \\ - doenças ósseas \\ - tratamento farmacológico \\ - hemangioma \\ - doenças da coluna vertebral}

\section{Abstract \\ Keywords \\ - bone diseases \\ - drug therapy \\ - hemangioma \\ - spinal diseases}

O hemangioma vertebral, um tumor vascular benigno, geralmente é assintomático e descoberto incidentalmente em exames de imagem. Quando sintomático, a apresentação mais frequente ocorre sob a forma de dorsalgia vaga de início insidioso e, em raros casos, pode estar associado a compressão radicular ou medular, causando déficit sensitivo e motor. Os autores relatam o caso de um homem de 33 anos, previamente hígido, com diagnósticos de hemangioma na coluna torácica em múltiplos níveis, no esterno, na escápula e nos arcos costais; todas as lesões eram sintomáticas e houve necessidade de intervenção cirúrgica, sendo que uma das lesões ao nível da coluna torácica evoluiu com compressão medular e déficit neurológico agudo, com necessidade de intervenção cirúrgica de urgência. Os hemangiomas intraósseos representam $<1 \%$ de todos os tumores ósseos, e a apresentação multifocal no esqueleto axial e apendicular apresenta poucos relatos. Na revisão bibliográfica, não foi encontrado outro caso de hemangioma intraósseo multifocal agressivo com tal apresentação, inclusive com sintomas neurológicos associados em um mesmo caso.

Vertebral hemangioma is a benign vascular tumor that is usually asymptomatic and is discovered incidentally on imaging. When symptomatic, the most frequent presentation occurs in the form of vague back pain of insidious onset and, in rare cases, may be associated with root or spinal compression, causing sensory and motor deficits. The authors report the case of a 33-year-old man, previously healthy, with a diagnosis of thoracic spine hemangioma at multiple levels, in the sternum, in the scapula and in the costal arches; all lesions were symptomatic, and surgical intervention was required; one of the lesions at the thoracic spine level evolved with spinal compression and acute neurological deficit, requiring urgent surgical intervention. Intraosseous hemangiomas represent $<1 \%$ of all bone tumors, having few reports of multifocal presentation in the axial and appendicular skeleton. In the literature review, no other case of aggressive multifocal intraosseous hemangioma with this presentation was found, including associated neurological symptoms in the same case. recebido

15 de Agosto de 2020

aceito

02 de Outubro de 2020
DOI https://doi.org/

10.1055/s-0040-1722585. ISSN 0102-3616. (c) 2021. Sociedade Brasileira de Ortopedia e Traumatologia. All rights reserved.

This is an open access article published by Thieme under the terms of the Creative Commons Attribution-NonDerivative-NonCommercial-License, permitting copying and reproduction so long as the original work is given appropriate credit. Contents may not be used for commercial purposes, or adapted, remixed, transformed or built upon. (https://creativecommons.org/ licenses/by-nc-nd/4.0/)

Thieme Revinter Publicações Ltda., Rua do Matoso 170, Rio de Janeiro, RJ, CEP 20270-135, Brazil 


\section{Introdução}

O hemangioma é uma lesão benigna, do grupo das lesões vasculares, constituída por vasos sanguíneos neoformados. Algumas dessas lesões são malformações, enquanto outras, em virtude do crescimento com características de neoplasia, são consideradas verdadeiros tumores benignos. ${ }^{1}$ As localizações mais comuns dos hemangiomas são o crânio, onde podem produzir a imagem clássica em "raios de sol", e o esqueleto axial, geralmente sem nenhuma sintomatologia clínica. ${ }^{1} \mathrm{Na}$ maioria das vezes, os hemangiomas não necessitam de tratamento, mas sim de seguimento periódico.

A idade média dos pacientes é de 40 anos, sendo mais frequente em mulheres, na proporção de $3: 2 .^{1,2}$ Na radiografia simples, os hemangiomas caracterizam-se por apresentar trabeculados verticais paralelos nos corpos vertebrais. A tomografia computadorizada (TC) revela a presença de trabéculas espessas e áreas radiotransparentes, e a ressonância magnética (RM) é altamente sensível e específica, apresentando sinais hiperintensos em T1 e T2. 1,3

Descrevemos um caso raro de hemangiomatose agressiva em um paciente jovem a fim de demonstrar a apresentação clínica, os exames de imagem, bem como a evolução do paciente com o tratamento realizado. Por fim, alertamos para o diagnóstico de hemangioma como diagnóstico diferencial em lesões ósseas múltiplas e, também, agressivas.

\section{Relato de Caso}

Paciente do sexo masculino, 33 anos, metalúrgico, previamente hígido, com história de dorsalgia crônica sem irradiação, RM de coluna torácica prévia com achado sugestivo de hemangioma em T2, T3, T4, T9 e L1 ( - Figura 1 ). O paciente evoluiu com piora da dorsalgia e irradiação bilateral para os membros inferiores. $O$ paciente foi submetido a uma nova RM após a piora dos sintomas, $\sim 1$ ano após o primeiro exame, sendo visualizado um aumento da lesão prévia em T3 com invasão do canal medular, ocasionando compressão medular (-Figura 2); o paciente foi então encaminhado ao nosso serviço.

Ao exame, o paciente apresentava parestesia dos membros inferiores, clônus presente e marcha atáxica. Devido à agressividade da lesão e aos sintomas neurológicos agudos, foram indicadas internação hospitalar e intervenção cirúrgica. Foi realizada descompressão medular associada a artrodese do segmento T2-T5. Constatou-se hemangioma do corpo vertebral pelo exame anatomopatológico. O paciente foi submetido a 20 sessões de radioterapia após a cirurgia e manteve acompanhamento semestral com boa evolução.

Os outros hemangiomas dos corpos vertebrais descritos se mantiveram com tratamento conservador e acompanhamento com exames de imagem, uma vez que não apresentaram crescimento nem sintomas (-Figura $\mathbf{3}$ ).

Dois anos mais tarde, o paciente apresentou queixa de dor importante em arcos costais à direita, realizado radiografia sem particularidades. Foi solicitada uma cintilografia óssea, a qual apresentou captação nos $4^{\circ}$ e $6^{\circ}$ arcos costais à direita. Foi realizado complemento com RM e TC, e foi indicada a ressecção

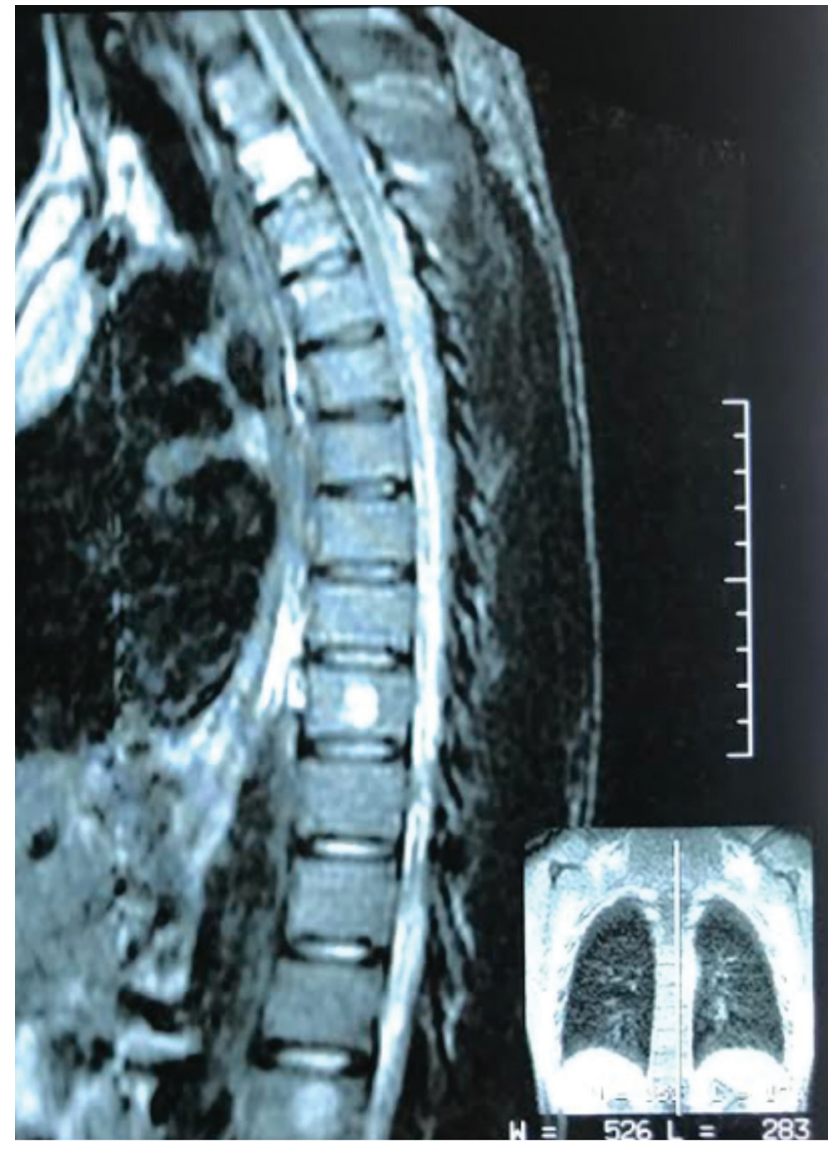

Fig. 1 Corte sagital de ressonância magnética da coluna dorsal demonstrando hemangiomas nos níveis T2,T3,T4,T9 e L1.

dos hemangiomas dos $4^{\circ}$ e $6^{\circ}$ arcos costais à direita, confirmados por exame anatomopatológico (- Figura 4A).

Durante o seguimento, 3 anos após a intervenção ao nível dos arcos costais, o paciente apresentou dor no ombro à direita. Foi realizada uma investigação, a qual diagnosticou uma lesão em escápula à direita (-Figura 5). Foi indicada ressecção, com o laudo anatomopatológico resultando em outro hemangioma.

No mesmo ano, o paciente apresentou queixa de dor torácica anterior, inclusive procurando atendimento de emergência devido à intensidade dos sintomas. Foi realizada uma RM, a qual apresentou uma lesão no esterno ( - Figura 4B). Foi indicada a ressecção, o material foi enviado para exame anatomopatológico e foi confirmado novo hemangioma.

\section{Discussão}

A apresentação difusa do hemangioma intraósseo é incomum e, quando relatada, costuma se apresentar em uma região, como em vários corpos vertebrais, mas não costuma se distribuir em diferentes sítios do corpo. ${ }^{1,4}$

As localizações mais comuns dos hemangiomas são o crânio e o esqueleto axial. Na coluna, a localização torácica é mais comum nas regiões média e inferior, seguidas da região lombar, ocorrendo mais raramente na região cervical. ${ }^{1,2}$

O tratamento do hemangioma intraósseo depende dos sintomas em cada região afetada. ${ }^{4}$ 


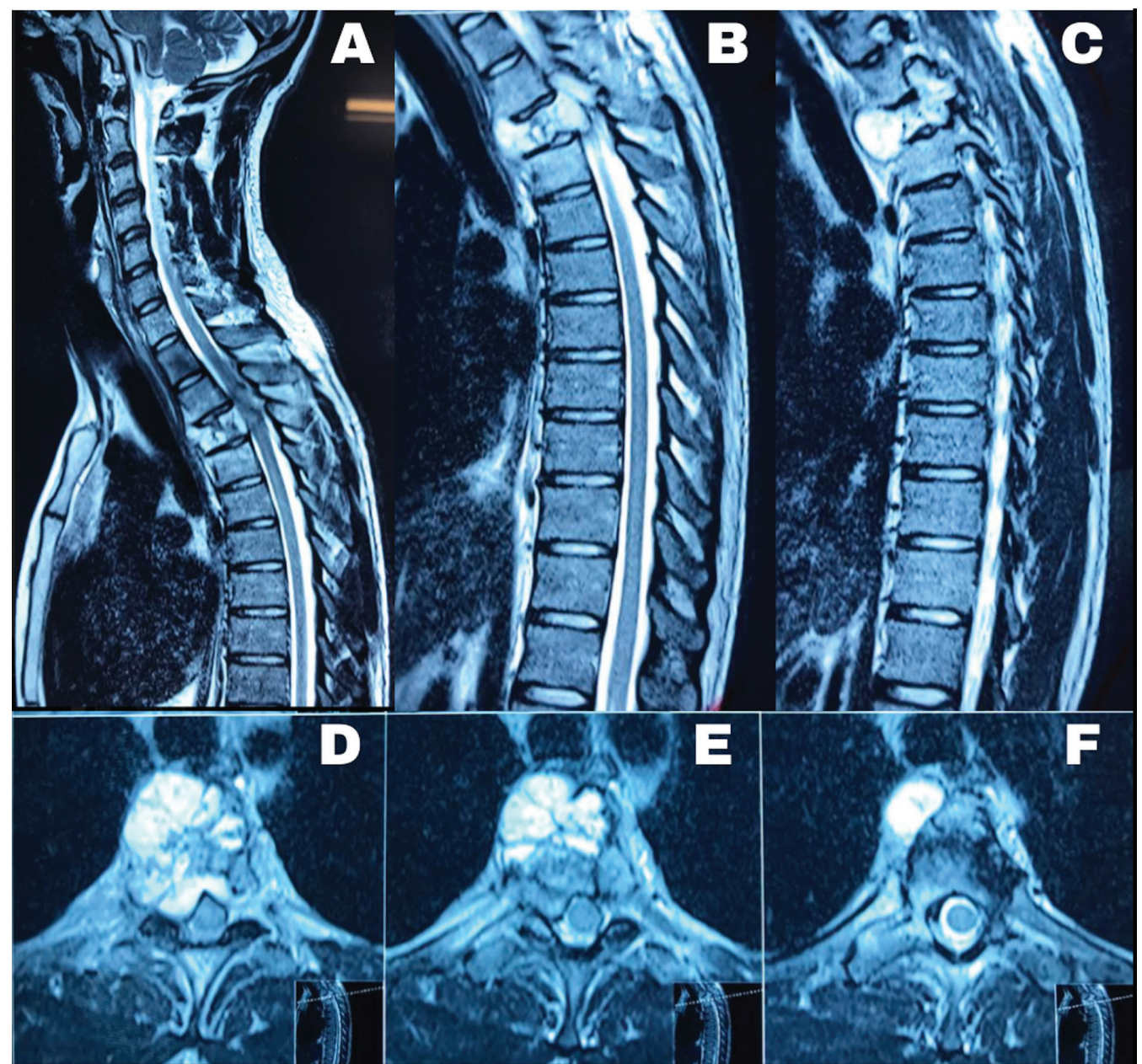

Fig. 2 Corte sagital de ressonância magnética (A,B e C) demonstrando aumento das dimensões da lesão no nível de T3 com invasão do canal medular; Corte axial (D,E e F) ao nível da lesão evidenciando dismorfismo medular e compressão do canal medular.

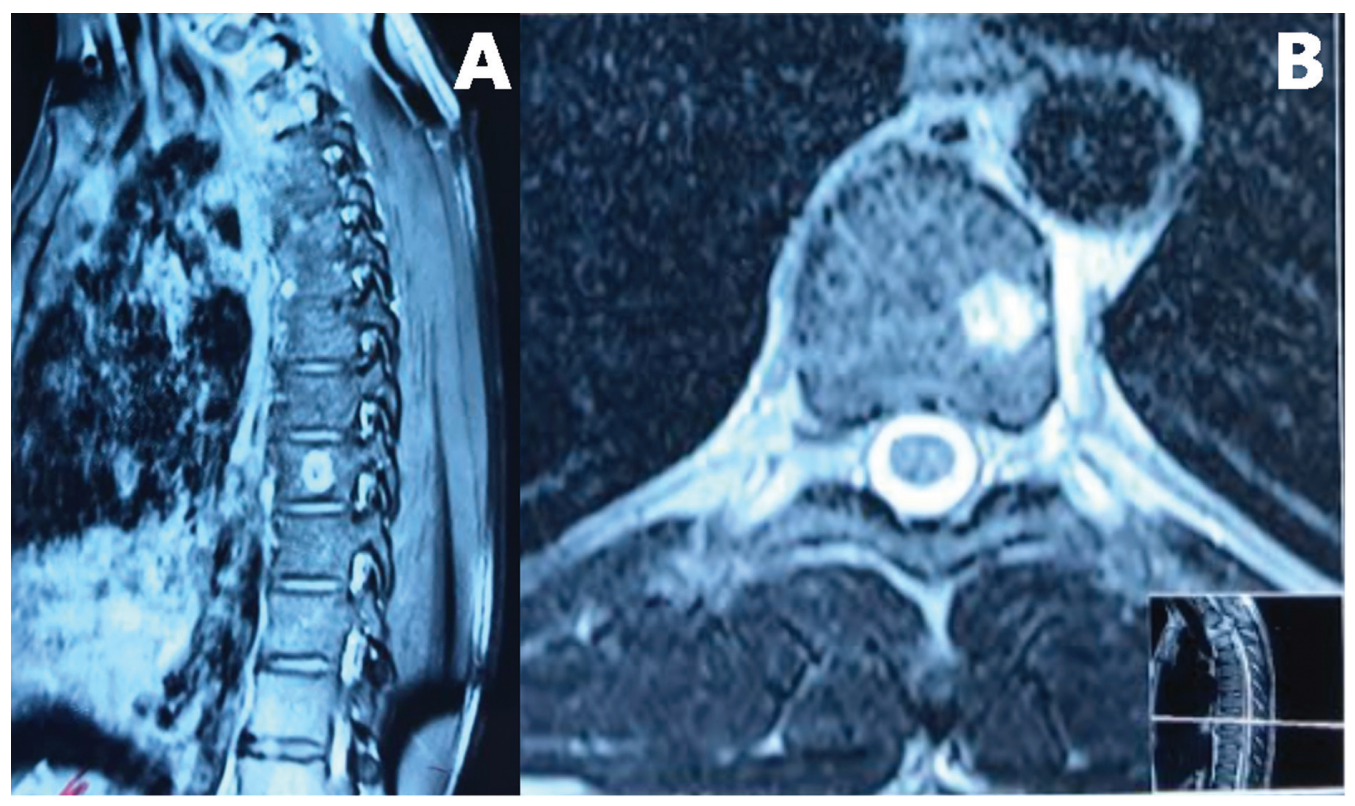

Fig. 3 Corte sagital (A) e axial (B) de ressonância magnética demonstrando hemangioma nodular no nível de T9. 


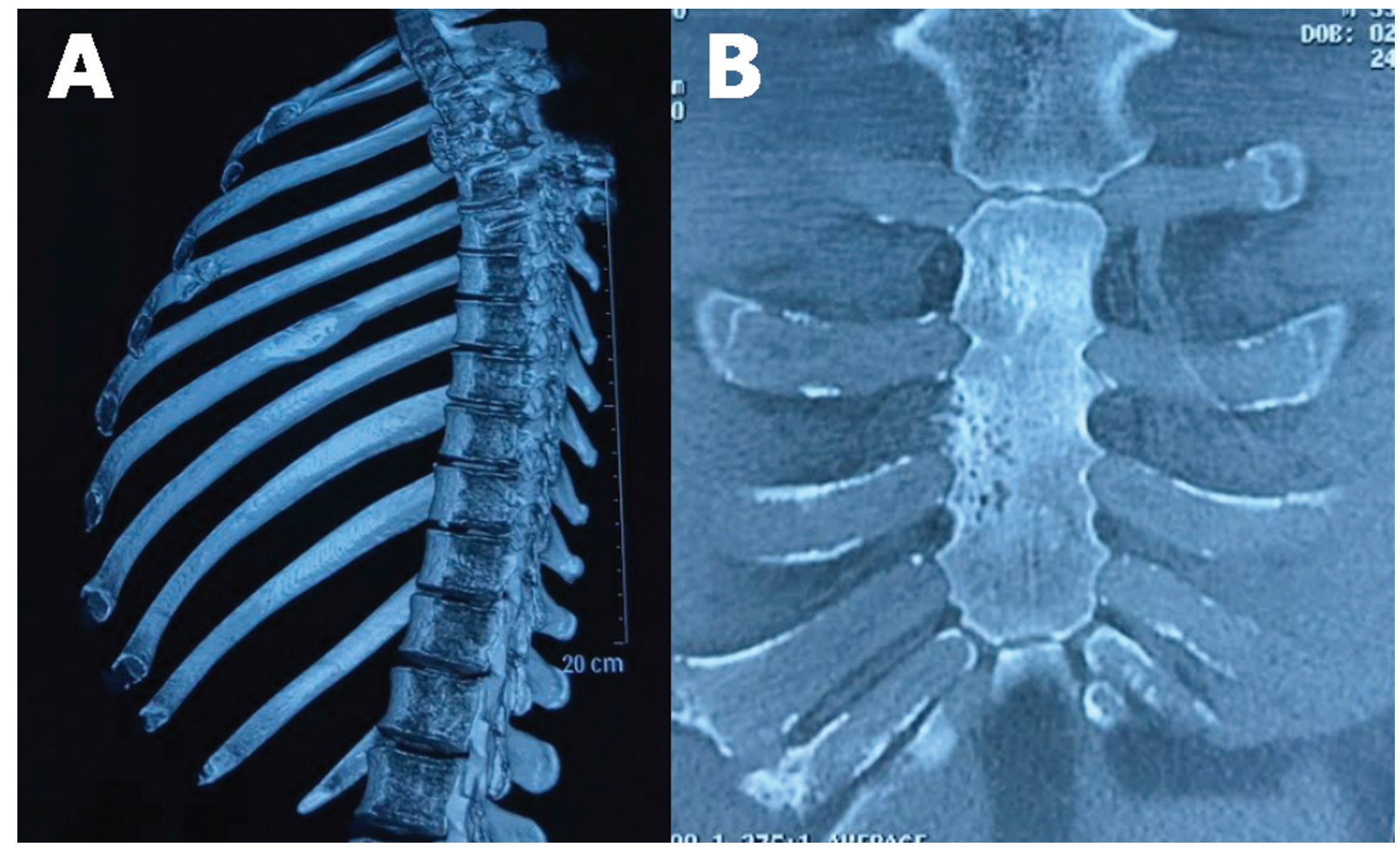

Fig. 4 Reconstrução por tomografia computadorizada (A) com hemangioma nos $4^{\circ}$ e $6^{\circ}$ arcos costais; tomografia computadorizada do esterno (B) demonstrando lesão inicial.
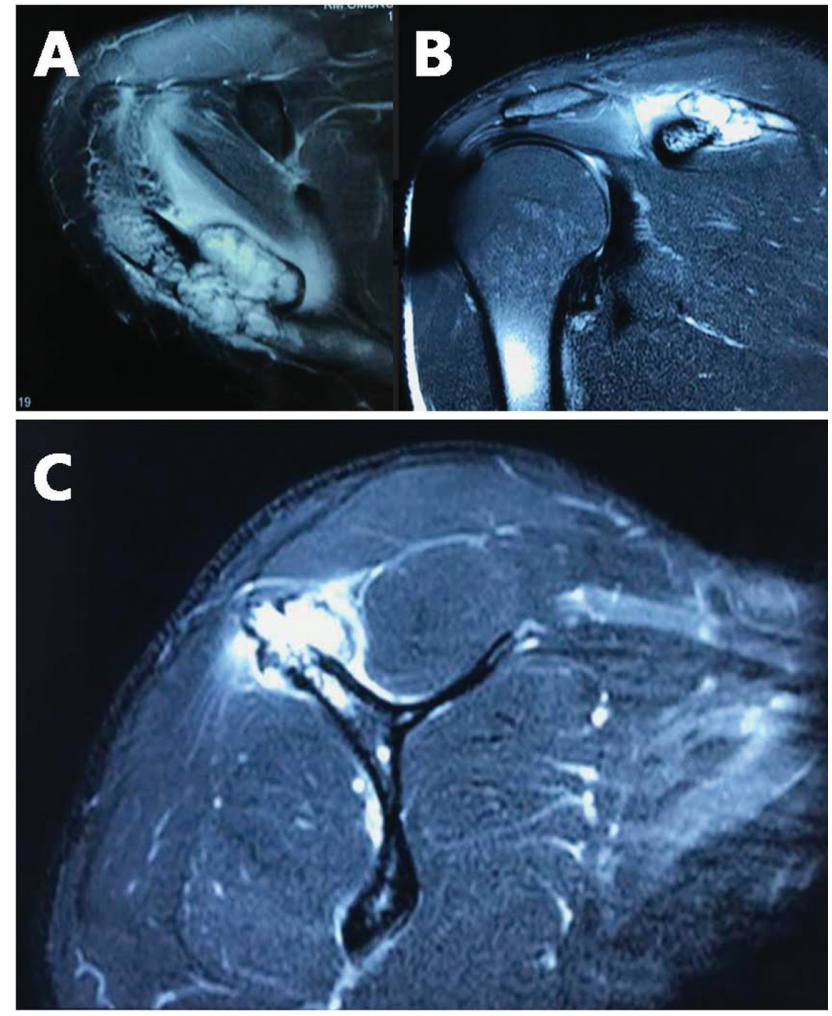

Fig. 5 Ressonância magnética, corte axial (A), coronal (B) e sagital (C) da escápula com lesão expansiva.

A maioria dos hemangiomas vertebrais é latente e não requer especificidades no tratamento; poucos casos evoluem com sintomas, sendo que, quando presentes, costumam se limitar à presença de dor. Contudo, em raros casos, heman- giomas podem se apresentar agressivos, com déficit neurológico decorrente de compressão medular. ${ }^{1,2,5-8}$

A forma evolutiva do presente caso demonstra lesões difusas, em paciente masculino, sintomáticas, agressivas e não concomitantes, com surgimento de novos hemangiomas durante o seguimento, não passíveis de tratamento conservador.

As modalidades terapêuticas são amplas: radioterapia, embolização arterial, ligadura dos vasos nutrientes, cirurgia decompressiva para coluna e ressecção do tumor. ${ }^{8,9}$ Nos casos de compressão medular, cirurgia decompressiva seguida ou não de radioterapia tem sido o tratamento de escolha. ${ }^{3,10} \mathrm{As}$ opções de tratamento podem ser usadas isoladamente ou associadas ${ }^{9}$, variando conforme os sintomas, a evolução do caso e dependendo da experiência do médico assistente. ${ }^{1}$

A vertebroplastia também é descrita como modalidade terapêutica para melhorar os sintomas de dor nos casos sem déficit neurológico, mas com menor benefício a longo prazo no alívio da dor. ${ }^{10}$

A radioterapia pode ser utilizada no tratamento dos hemangiomas vertebrais de forma exclusiva ou associada à cirurgia. $^{5}$

Quadros associados a dorsalgia e sem déficits neurológicos podem ser conduzidos mediante observação periódica e tratamento clínico. Em casos refratários, radioterapia exclusiva pode ser uma opção para controle da dor através da necrose vascular e/ou um efeito anti-inflamatório. ${ }^{5}$

\section{Nota}

Trabalho desenvolvido no Hospital são Lucas, Serviço de Ortopedia e Traumatologia, Pontifícia Universidade Católica do Rio Grande do Sul, Porto Alegre, RS, Brasil. 


\section{Conflito de Interesses}

Os autores declaram não haver conflito de interesses.

\section{Referências}

1 Oliveira RP, Rodrigues NR, França AF, et al. Relato de quatro casos de hemangioma de coluna vertebral com evolução atípica. Rev Bras Ortop 1996;31(02):119-124

2 Sari H, Uludag M, Akarirmak U, Ornek NI, Gun K, Gulsen F. Aggressive vertebral hemangioma as a rare cause of myelopathy. J Back Musculoskeletal Rehabil 2014;27(02):125-129

3 Castro DG, Lima RP, Maia MAC, et al. Hemangioma vertebral sintomático tratado com radioterapia exclusiva: relato de caso e revisão da literatura. Radiol Bras 2002;35(03):179-181

4 Yao K, Tang F, Min L, Zhou Y, Tu C. Multifocal intraosseous hemangioma: A case report. Medicine (Baltimore) 2019;98(02):e14001

5 Jiang L, Liu XG, Yuan HS, et al. Diagnosis and treatment of vertebral hemangiomas with neurologic deficit: a report of 29 cases and literature review. Spine J 2014;14(06):944-954
6 Chen HI, Heuer GG, Zaghloul K, Simon SL, Weigele JB, Grady MS. Lumbar vertebral hemangioma presenting with the acute onset of neurological symptoms. Case report. J Neurosurg Spine 2007;7 (01):80-85

7 Dickerman RD, Bennett MT. Acute spinal cord compression caused by vertebral hemangioma. Spine J 2005;5(05):582-584, author reply 584

8 Hu W, Kan SL, Xu HB, Cao ZG, Zhang XL, Zhu RS. Thoracic aggressive vertebral hemangioma with neurologic deficit: A retrospective cohort study. Medicine (Baltimore) 2018;97(41): e12775

9 Delabar V, Bruneau M, Beuriat PA, et al. [The efficacy of multimodal treatment for symptomatic vertebral hemangiomas: A report of 27 cases and a review of the literature]. Neurochirurgie 2017;63(06):458-467

10 Acosta FL Jr, Dowd CF, Chin C, Tihan T, Ames CP, Weinstein PR. Current treatment strategies and outcomes in the management of symptomatic vertebral hemangiomas. Neurosurgery 2006;58 (02):287-295, discussion 287-295 